\title{
ARTIGO
}

\section{ANÁLISE DO DESEMPENHO DO MOTOR DE INDUÇÃO TRIFÁSICO POR MEIO DE ENSAIOS RECOMENDADOS EM NORMA TÉCNICA E SIMULAÇÃO COMPUTACIONAL ${ }^{1}$}

\author{
Vinícius Marcos Pinheiro \\ Luciano Coutinho Gomes \\ Cássio Alves de Oliveira
}

\begin{abstract}
RESUMO
Este trabalho apresenta um estudo realizado para a determinação dos parâmetros do circuito equivalente de um motor de indução trifásico a partir de ensaios recomendados na norma NBR17094-3:2018 da Associação Brasileira de Normas Técnicas (ABNT), além da verificação do seu desempenho por meio da variação de carga em seu eixo, utilizando para isso, simulação computacional. Os parâmetros obtidos durante a realização dos ensaios são utilizados em software para a análise do fator de potência em diferentes situações de carga.
\end{abstract}

Palavras-chaves: Motor de indução trifásico. NBR17094-3:2018. Parâmetros. Simulação computacional.

\section{INTRODUÇÃO}

Os motores de indução são máquinas elétricas cuja invenção é datada do ano de 1888, sendo a patente original pertencente a Nikola Tesla (DA SILVEIRA, 2012; MARQUES; 2012). Tais motores, portanto, passaram a ser empregados fortemente na indústria, para tornar os processos mais práticos e rápidos. Geralmente, a principal carga elétrica de uma indústria está concentrada neste tipo de equipamento, o que mostra o quão importante é sua análise, para verificar alguns tipos de fenômenos que podem estar acometendo o sistema elétrico, principalmente, no ambiente interno à indústria, como exemplo, o baixo fator de potência, que pode até acarretar multas.

Contudo, para a análise de qualquer tipo de equipamento, é crucial seguir uma diretriz para a aquisição dos parâmetros do mesmo. Desta maneira, as normas surgem com esse

\footnotetext{
${ }^{1}$ Como inserir este artigo: PINHEIRO, Vinícius Marcos; GOMES, Luciano Coutinho; OLIVEIRA, Cássio Alves de. Análise do desempenho do motor de indução trifásico por meio de ensaios recomendados em norma técnica e simulação computacional. ForScience: revista científica do IFMG, Formiga, v. 7, n. 1, e00362, jan./jun. 2019. DOI: 10.29069/forscience.2019v7n1.e362.
}

\footnotetext{
${ }^{2}$ Autor para correspondência: Vinícius Marcos Pinheiro. E-mail: viniciusmarcospinheiro@hotmail.com.
} 
objetivo, ou seja, elas visam destacar como e quando os procedimentos devem ser realizados, os cuidados que devem ser tomados e como realizar os devidos cálculos para a determinação dos parâmetros do equipamento em estudo.

Por meio dos parâmetros bem definidos, a utilização dos recursos computacionais possibilita o estudo do comportamento do motor em diversas situações, muitas das quais não seriam passíveis de serem realizadas na prática.

Sendo assim, o objetivo do presente trabalho foi efetuar o levantamento dos parâmetros do circuito equivalente de um motor de indução trifásico a partir das recomendações colocadas pela NBR17094-3:2018. Posteriormente, com os dados dos parâmetros em mãos, realizou-se uma análise, por meio de simulação computacional, do comportamento do motor em alguns regimes de operação, observando os valores de fator de potência para cada caso.

\section{METODOLOGIA APLICADA E MATERIAIS UTILIZADOS}

Os parâmetros do motor de indução trifásico necessários para a respectiva análise do mesmo, serão obtidos por meio de ensaios práticos, seguindo os passos descritos pela norma brasileira NBR17094-3:2018. Tal norma trata a respeito das máquinas elétricas girantes, mais especificamente, dos ensaios de motores de indução trifásico.

\subsection{Circuito equivalente do motor de indução trifásico}

O circuito equivalente do motor de indução trifásico, como é definido por Fitzgerald (2006), está representado pela Figura 1. O respectivo circuito é uma representação por fase, ou seja, corresponde a um circuito equivalente monofásico, sendo, as demais fases, obtidas defasando a corrente e a tensão de $\pm 120^{\circ}$.

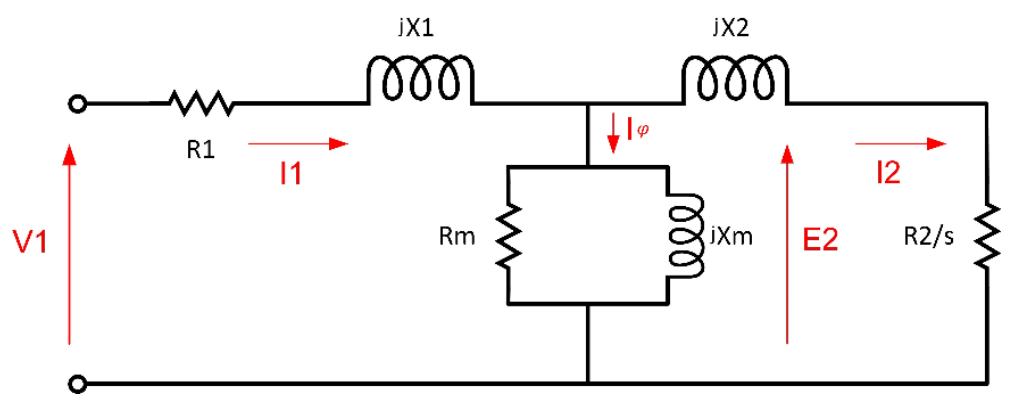

Figura 1 - Circuito equivalente do motor de indução trifásico Fonte: Elaborada pelo autor. 
Na qual:

- $\hat{\mathrm{I}}_{1}-$ Corrente do estator $[\mathrm{A}]$;

- $V_{1}$-Tensão no terminal do estator [V];

- $X_{1}-$ Reatância de dispersão do estator $[\Omega]$;

- $R_{1}$ - Resistência do estator $[\Omega]$;

- $R_{2}-$ Resistência do rotor $[\Omega]$;

- $X_{2}-$ Reatância de dispersão do rotor $[\Omega]$;

- $\hat{\mathrm{I}}_{\varphi}-$ Corrente de excitação [A];

- $\hat{\mathrm{I}}_{2}$ - Corrente de carga [A];

- $E_{2}-$ Força contra eletromotriz [V];

- $R_{m}$ - Resistência de perda no núcleo $[\Omega]$;

- $X_{m}$-Reatância de magnetização [ $\left.\Omega\right]$.

Para obter os parâmetros do circuito equivalente, devem ser realizados três ensaios seguindo a NBR17094-3:2018, os quais são: ensaio para determinação da resistência do enrolamento do estator, ensaio de rotor bloqueado e ensaio a vazio.

\subsection{Fonte Programável}

Para a realização dos ensaios citados anteriormente, utiliza-se uma fonte programável, representada pela Figura 2, da AMETEK Programmable Power®, cujo modelo é o CSW5550. A mesma é empregada para alimentar o motor de indução trifásico.

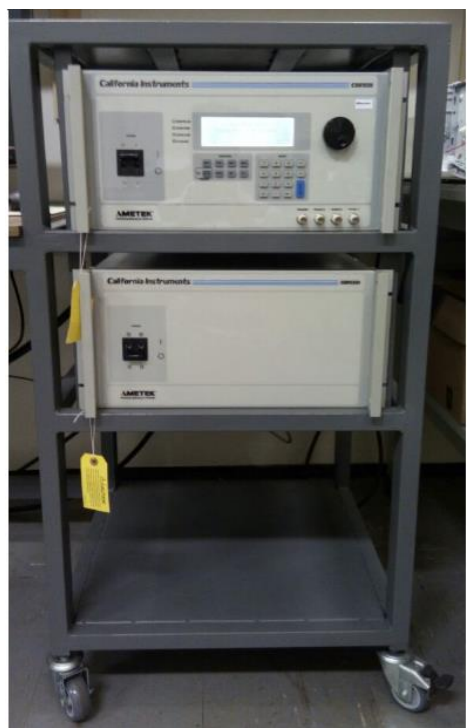

Figura 2 - Fonte programável Fonte: Elaborada pelo autor.

ForSci.: r. cient. IFMG campus Formiga, Formiga, v. 7, n. 1, e00362, jan./jun. 2019. 
Este equipamento possibilita mensurar diversas grandezas, como: tensão, corrente, potência ativa e aparente, defasamento angular e fator de potência.

\subsection{Motor de indução trifásico}

O motor de indução trifásico a ser analisado é da fabricante WEG®, sendo seus dados de placa apresentados no Quadro 1.

\begin{tabular}{|c|c|}
\hline \multicolumn{2}{|c|}{$\begin{array}{c}\text { Motor de indução trifásico gaiola de esquilo WEG® } \\
\text { Categoria } \mathrm{N}-60 \mathrm{~Hz} \text { - IP55 - F.S 1,15 - S1 - Classe Isol F }\end{array}$} \\
\hline Potência $(K W / C V)$ & $2,2 / 3,0$ \\
\hline Tensão $(\Delta / Y)$ & $220 / 380 \mathrm{~V}$ \\
\hline Corrente $(\Delta / Y)$ & $8,39 / 4,86 \mathrm{~A}$ \\
\hline Fator de Potência & 0,84 \\
\hline Velocidade & $3450 \mathrm{rpm}$ \\
\hline$I_{p} / I_{n}$ & 6,7 \\
\hline
\end{tabular}

Apesar de haver dois tipos de ligações nas quais as bobinas do estator possam ser arranjadas, tratando-se das ligações estrela e delta, optou-se por esse último arranjo, o qual está representado pela Figura 3, devido a limitação de tensão. Os terminais do motor foram identificados respectivamente como fases A, B e C.

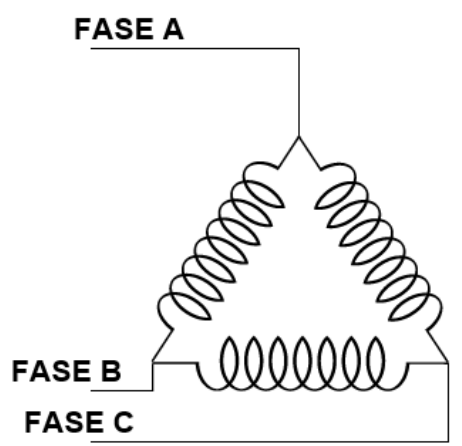

Figura 3 - Arranjo de ligação em delta Fonte: Elaborada pelo autor 


\subsection{Simulação computacional}

A simulação computacional do motor de indução trifásico é realizada através de um modelo já presente no programa PSIM®. O intuito da simulação é estudar alguns regimes de operação que serão, posteriormente, definidos.

\subsection{Características dos ensaios conforme a NBR17094-3:2018}

A norma NBR17094-3:2018 descreve os ensaios a serem realizados, como são apresentados a seguir.

\subsubsection{Ensaio para determinação da resistência do enrolamento do estator}

Segundo a NBR17094-3:2018, pode-se determinar a resistência de enrolamento a partir de dois métodos, os quais são:

- Método da tensão e corrente;

- Método da ponte.

A medição da resistência de enrolamento é realizada aplicando-se corrente contínua nos terminais do motor de indução. Indiretamente, pode-se utilizar a medição da resistência de enrolamento para verificar a integridade do mesmo, averiguando a existência de curtos circuitos nas espiras ou contatos em condições não ideais, por meio da comparação dos valores de resistências medidos e os fornecidos pelo fabricante.

De posse dos valores de tensão e corrente, pode-se determinar o valor da resistência a partir da Lei de Ohm, definida pela Equação (1).

$$
R I=V
$$

Na qual:

- $R$ - Resistência $[\Omega]$;

- $I$ - Corrente $[\mathrm{A}]$;

- $V$ - Tensão [V]. 
Por fim, é importante corrigir cada resistência do enrolamento para uma temperatura base definida pela norma, que é a temperatura ambiente de $25^{\circ} \mathrm{C}$, para, posteriormente, calcular a média aritmética das mesmas. Tal correção é realizada por meio da Equação (2).

Sendo:

$$
R_{s}=\frac{R_{t}\left(t_{s}+k\right)}{\left(t_{t}+k\right)}
$$

- $R_{S}$ - Resistência do enrolamento corrigida para uma temperatura especificada, $t_{s}$;

- $t_{s}$ - Temperatura especificada para correção da resistência, em graus Celsius;

- $R_{t}$ - Resistência do enrolamento obtida no ensaio à temperatura $t_{t}$;

- $t_{t}$ - Temperatura do enrolamento no momento da medição da resistência, em graus Celsius.

- $k$-Constante baseada no material condutor do enrolamento. (Para cobre eletrolítico com $100 \%$ de condutividade $\mathrm{K}=234,5$. Para alumínio com condutividade em volume de $62 \%, \mathrm{~K}=225)$.

Vale ressaltar, como a norma recomenda, que as medições em cada ensaio devem possuir uma duração máxima de um minuto, devido à elevação de temperatura do enrolamento do estator quando o ensaio é executado.

É importante salientar, que as medições das correntes e tensões, são efetuadas via sistema operacional da própria fonte de alimentação, presente em um microcomputador. Sendo assim, para cada nível de tensão estipulado, é realizada a coleta de uma medição para cada fase e medida a temperatura do enrolamento.

\subsubsection{Ensaio de rotor bloqueado}

A realização do ensaio de rotor bloqueado visa a determinação das reatâncias de dispersão do motor de indução trifásico, bem como a resistência do rotor, onde, mantendo-se o eixo do motor completamente bloqueado, aplica-se tensão reduzida, a qual é elevada gradativamente de tal forma a se obter corrente nominal.

A NBR17094-3:2018 estabelece algumas diretrizes a serem seguidas antes da realização do presente ensaio, as quais são: 
- O meio mecânico de bloqueio do rotor deve ter rigidez adequada para evitar possível risco ao pessoal ou dano ao equipamento;

- O sentido de rotação deve ser estabelecido antes do ensaio;

- O motor deve estar aproximadamente à temperatura ambiente antes do início do ensaio.

Um ponto importante para se obter as medidas é com relação à temperatura do motor, que não deve ultrapassar o limite de elevação de temperatura nominal somado de $40^{\circ} \mathrm{C}$, para que não ocorra danificação no respectivo equipamento.

\subsubsection{Ensaio a vazio}

A realização do ensaio a vazio ocorre sob tensão e frequência nominal, sem carga, ou seja, com o eixo totalmente livre. O objetivo do ensaio a vazio pode ser resumido na determinação dos parâmetros do ramo magnetizante.

É recomendado pela NBR17094-3:2018 que as medições só sejam realizadas após a estabilização da potência de entrada, pois, assim, assegura-se que o valor da perda por atrito seja obtido de forma mais precisa, visto que o excesso de graxa nos mancais e nas partes móveis do motor, acabam interferindo na variação da potência de entrada até que a condição de operação seja estabilizada. Segundo a respectiva norma, o ponto de estabilização é alcançado quando a potência absorvida em vazio não variar mais que $3 \%$ entre duas leituras realizadas em um intervalo de tempo de 30 minutos.

\subsubsection{Determinação dos parâmetros do circuito equivalente}

Os cálculos dos parâmetros do circuito equivalente são realizados a partir do ensaio de rotor bloqueado e do ensaio a vazio. A determinação dos parâmetros inicia-se a partir da Figura 4, que representa o circuito equivalente do motor de indução trifásico para o ensaio de rotor bloqueado. 


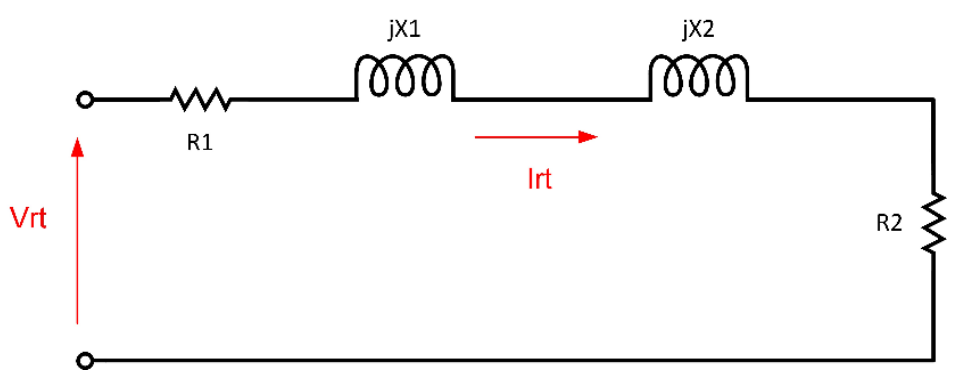

Figura 4 - Circuito equivalente para o ensaio de rotor bloqueado Fonte: Elaborada pelo autor

Na qual:

- $V_{r t}-$ Tensão por fase de rotor bloqueado [V];

- $I_{r t}-$ Corrente por fase de rotor bloqueado [A];

- $R_{1}-$ Resistência do estator $[\Omega]$;

- $X_{1}-$ Reatância de dispersão do estator $[\Omega]$;

- $R_{2}-$ Resistência do rotor $[\Omega]$;

- $X_{2}-$ Reatância de dispersão do rotor $[\Omega]$.

Primeiramente, deve-se ter em mente que os cálculos dos parâmetros são realizados por fase, logo, é importante atentar-se ao utilizar os valores de corrente, tensão e potência. Outro ponto importante a ser comentado, é que foi utilizado o valor da média aritmética das medidas obtidas nas três fases do motor.

A Equação (3) permite calcular a resistência de rotor bloqueado a partir dos dados obtidos durante o ensaio à temperatura registrada.

$$
R_{r t}=\frac{P_{r t}}{I_{r t}^{2}}
$$

Na qual:

- $R_{r t}-$ Resistência do enrolamento de rotor bloqueado;

- $P_{r t}$ - Potência ativa por fase de rotor bloqueado;

- $I_{r t}$ - Corrente por fase de rotor bloqueado.

Posteriormente, pode-se calcular a impedância de rotor bloqueado através da Equação (4).

$$
Z_{r t}=\frac{V_{r t}}{I_{r t}}
$$


Na qual:

- $Z_{r t}$ - Impedância de rotor bloqueado;

- $V_{r t}-$ Tensão de fase de rotor bloqueado.

De posse dos valores da resistência e da reatância de rotor bloqueado, torna-se possível determinar o valor da reatância de rotor bloqueado por meio da Equação (5).

$$
X_{r t}=\sqrt{Z_{r t}^{2}-R_{r t}^{2}}
$$

O próximo passo é separar as reatâncias de dispersão tanto do rotor como do estator, da reatância de rotor bloqueado. Para tal, utiliza-se como auxílio o Quadro 2.

\begin{tabular}{|c|c|c|c|}
\hline \multicolumn{2}{|c|}{} & \multicolumn{2}{|c|}{$\begin{array}{c}\text { Fração de } \\
\boldsymbol{X}_{\mathbf{1}}+\boldsymbol{X}_{\mathbf{2}}\end{array}$} \\
\hline $\begin{array}{c}\text { Classe } \\
\text { de motor }\end{array}$ & $\boldsymbol{X}_{\mathbf{1}}$ & $\boldsymbol{X}_{\mathbf{2}}$ \\
\hline A & $\begin{array}{c}\text { Descrição } \\
\text { partida normal }\end{array}$ & 0,5 & 0,5 \\
\hline B & $\begin{array}{c}\text { Conjugado de partida normal, corrente de } \\
\text { partida baixa }\end{array}$ & 0,4 & 0,6 \\
\hline C & $\begin{array}{c}\text { Conjugado de partida elevado, corrente de } \\
\text { partida baixa }\end{array}$ & 0,3 & 0,7 \\
\hline
\end{tabular}

Quadro 2 - Fração de X1 e X2 com relação à classe do motor de indução Fonte: Fitzgerald (2006).

\begin{tabular}{|c|c|c|c|}
\hline \multicolumn{2}{|c|}{} & \multicolumn{2}{|c|}{$\begin{array}{c}\text { Fração de } \\
X_{\mathbf{1}}+\boldsymbol{X}_{\mathbf{2}}\end{array}$} \\
\hline $\begin{array}{c}\text { Classe } \\
\text { de motor }\end{array}$ & Descrição & $\boldsymbol{X}_{\mathbf{1}}$ & $\boldsymbol{X}_{\mathbf{2}}$ \\
\hline $\mathrm{D}$ & $\begin{array}{c}\text { Conjugado de partida elevado, } \\
\text { escorregamento elevado }\end{array}$ & 0,5 & 0,5 \\
\hline $\begin{array}{c}\text { Rotor } \\
\text { Bobinado }\end{array}$ & $\begin{array}{c}\text { Desempenho varia segundo a resistência do } \\
\text { rotor }\end{array}$ & 0,5 & 0,5 \\
\hline
\end{tabular}

Quadro 3 - Continuação - Fração de X1 e X2 com relação à classe do motor de indução Fonte: Fitzgerald (2006).

Por fim, é possível, ainda, dividir a resistência de rotor bloqueado nas resistências de rotor e de estator. Vale destacar que, como o motor possui um rotor de gaiola, não será 
possível determinar diretamente o valor da resistência do rotor. Contudo, há como estimar o valor de tal resistência, por meio da Equação (6).

$$
R_{r t}=R_{1}+R_{2}
$$

De posse dos dados obtidos durante o ensaio a vazio, pode-se realizar os cálculos dos parâmetros do ramo magnetizante. Para tal, utiliza-se como auxílio o circuito equivalente apresentado na Figura 5.

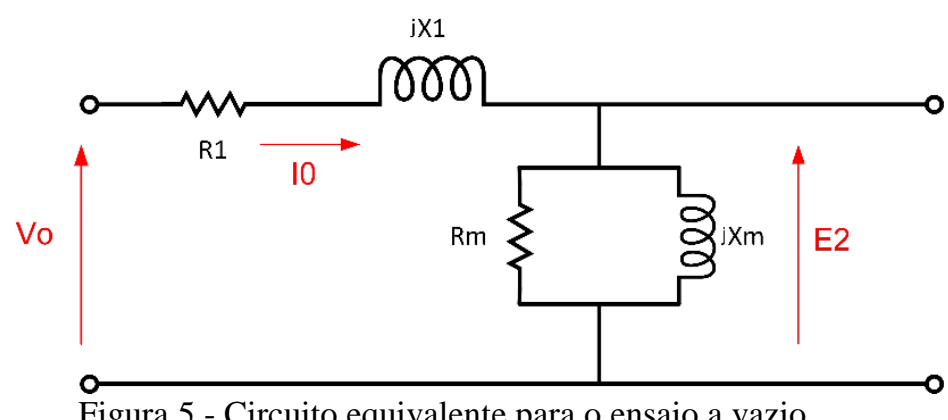

Figura 5 - Circuito equivalente para o ensaio a vazio

Fonte: Elaborada pelo autor

Na qual:

- $V_{o}$-Tensão por fase a vazio [V];

- $I_{O}$-Corrente por fase a vazio $[\mathrm{A}]$;

- $E_{2}-$ Força contra eletromotriz [V];

- $R_{m}-$ Resistência de perdas no núcleo $[\Omega]$;

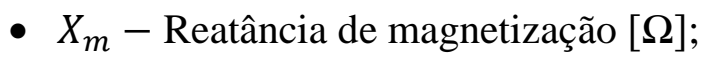

- $R_{1}-$ Resistência do estator $[\Omega]$;

- $X_{1}-$ Reatância de dispersão do estator $[\Omega]$.

Primeiramente, deve-se calcular a tensão que está sobre o ramo magnetizante para poder determinar o valor da reatância de magnetização e da resistência de perdas no núcleo, o que pode $\quad$ ser realizado por meio da Equação (7). Vale ressaltar que o valor da resistência do enrolamento do estator $\left(R_{1}\right)$ deve ser corrigida para a respectiva temperatura do ensaio a vazio.

$$
E_{2}=V_{o}-\left(\sqrt{\left(R_{1}\right)^{2}+\left(X_{1}\right)^{2}}\right) I_{o}
$$


Posteriormente, deve-se determinar os valores das correntes que circulam através da resistência de perda no núcleo e da reatância de magnetização, que podem ser calculadas, respectivamente, pelas Equações (8) e (9).

$$
\begin{gathered}
I_{c}=I_{o} \cos \varphi \\
I_{m}=I_{o} \operatorname{sen} \varphi
\end{gathered}
$$

Por fim, pela Lei de Ohm, pode-se determinar o valor da resistência e da reatância do ramo magnetizante.

\section{RESULTADOS}

Como resultados principais, o trabalho apresenta a obtenção dos parâmetros do circuito equivalente e as simulações computacionais, ambos descritos a seguir.

\subsection{Determinação dos parâmetros do circuito equivalente}

Conforme mencionado anteriormente, todos os ensaios realizados seguem a NBR17094-3:2018 e são apresentados a seguir.

\subsubsection{Ensaio para determinação da resistência do enrolamento do estator}

O procedimento para realização da medição da resistência do enrolamento de campo é apresentado na seção 2.5.1. Neste trabalho optou-se pelo método da tensão e corrente, em razão da fonte programável possibilitar, de forma fácil, tal aplicação.

São estipulados três níveis de tensão contínua, de modo que a corrente não ultrapasse $15 \%$ do valor nominal no enrolamento (no caso 5,589 amperes por fase), como é estabelecido pela norma. Por conseguinte, tais tensões são aplicadas em cada terminal do motor trifásico em estudo, separadamente, de modo a obter os valores das correntes e poder, assim, determinar a resistência do enrolamento do estator.

As Tabelas 1, 2 e 3 indicam os valores obtidos durante as medições, para as fases A, B e C, respectivamente, bem como os valores das resistências já corrigidas por meio da Equação (2). 
Tabela 1 - Medições realizadas para a fase A

\begin{tabular}{ccccc}
\hline \multicolumn{5}{c}{ Circuito da Fase A } \\
\hline $\mathbf{V}(\mathbf{V})$ & $\mathbf{I}(\mathbf{A})$ & $\begin{array}{c}\boldsymbol{R}_{\text {circuito- } \boldsymbol{A}} \\
(\boldsymbol{\Omega})\end{array}$ & $\begin{array}{c}\text { Temperatura } \\
\text { de ensaio }\left({ }^{\circ} \mathbf{C}\right)\end{array}$ & $\begin{array}{c}\boldsymbol{R}_{\text {circuito- }} \\
(\mathbf{\Omega}) \text { à 25 }\end{array}$ \\
\hline 3,76 & 1,72 & 2,186 & 25 & 2,186 \\
6,60 & 3,02 & 2,185 & 27 & 2,168 \\
9,18 & 4,14 & 2,217 & 27 & 2,200 \\
\hline
\end{tabular}

Fonte: Elaborada pelo autor

Tabela 2 - Medições realizadas para a fase B

\begin{tabular}{ccccc}
\hline \multicolumn{5}{c}{ Circuito da Fase B } \\
\hline $\mathbf{V}(\mathbf{V})$ & $\mathbf{I}(\mathbf{A})$ & $\begin{array}{c}\boldsymbol{R}_{\text {circuito-B }} \\
(\boldsymbol{\Omega})\end{array}$ & $\begin{array}{c}\text { Temperatura } \\
\text { de ensaio }\left({ }^{\circ} \mathbf{C}\right)\end{array}$ & $\begin{array}{c}\boldsymbol{R}_{\text {circuito-B }} \\
(\boldsymbol{\Omega}) \text { à 25}\end{array}$ \\
\hline 4,06 & 1,86 & 2,182 & 28 & 2,157 \\
6,63 & 3,02 & 2,195 & 28 & 2,169 \\
9,19 & 4,16 & 2,209 & 29 & 2,175
\end{tabular}

Fonte: Elaborada pelo autor

Tabela 3 - Medições realizadas para a fase $\mathrm{C}$

\begin{tabular}{ccccc}
\hline \multicolumn{5}{c}{ Circuito da Fase C } \\
\hline $\mathbf{V}(\mathbf{V})$ & $\mathbf{I}(\mathbf{A})$ & $\begin{array}{c}\boldsymbol{R}_{\text {circuito- } \boldsymbol{C}} \\
(\boldsymbol{\Omega})\end{array}$ & $\begin{array}{c}\text { Temperatura } \\
\text { de ensaio }\left({ }^{\circ} \mathbf{C}\right)\end{array}$ & $\begin{array}{c}\boldsymbol{R}_{\text {circuito-C }} \\
(\boldsymbol{\Omega}) \mathbf{a} \mathbf{2 5}\end{array}$ \\
\hline 4,41 & 1,98 & 2,227 & 30 & 2,184 \\
6,62 & 2,97 & 2,228 & 32 & 2,169 \\
9,18 & 4,07 & 2,255 & 36 & 2,163 \\
\hline
\end{tabular}

Fonte: Elaborada pelo autor

A Tabela 4 indica a média das resistências para cada fase, já corrigidas com relação à temperatura ambiente, empregando-se Equação (2).

Tabela 4 - Resistência média dos enrolamentos do estator por fase à $25^{\circ} \mathrm{C}$

\begin{tabular}{ccc}
\hline \multicolumn{2}{c}{ Resistência média dos enrolamentos do estator por fase à $25^{\circ} \mathbf{C}$} \\
\hline Fase A & Fase B & Fase C \\
\hline $2,184 \Omega$ & $2,167 \Omega$ & $2,172 \Omega$
\end{tabular}

Fonte: Elaborada pelo autor

Aplicando a média aritmética nos três valores encontrados, presentes na Tabela 4, é determinado o valor médio da resistência do enrolamento do estator à temperatura de $25^{\circ} \mathrm{C}$.

$$
R_{\text {média }_{\text {estator }}}=\frac{2,184+2,167+2,172}{3}=2,174 \Omega
$$


A medição da temperatura foi realizada via termopar, o qual foi instalado na parte interna do motor, mais especificamente no enrolamento do estator.

\subsubsection{Ensaio de rotor bloqueado}

Primeiramente, deve-se atentar para a elevação de temperatura, como foi exposto na seção 2.5.2. A temperatura máxima para este motor será de $155^{\circ} \mathrm{C}$, já que o mesmo é da classe térmica de isolamento $\mathrm{F}$.

O procedimento para realização do ensaio de rotor bloqueado é o descrito na seção 2.5.2. A Tabela 5 indica os valores obtidos, para cada fase.

Tabela 5 - Medições obtidas durante o ensaio de rotor bloqueado

\begin{tabular}{cccc}
\hline Grandeza medida & FASE A & FASE B & FASE C \\
\hline Tensão (Vrms) & 43,3 & 43,3 & 43,3 \\
Corrente (Arms) & 8,39 & 8,59 & 8,58 \\
Fase $(\boldsymbol{\phi})$ & 0 & 240,3 & 120,5 \\
Potência (kW) & 0,1 & 0,11 & 0,1 \\
Potência Aparente & 0,21 & 0,215 & 0,214 \\
$\quad$ (kVA) & 0,49 & 0,5 & 0,48 \\
Fator de Potência & 0,49 &
\end{tabular}

A temperatura registrada no interior do motor foi de $36^{\circ} \mathrm{C}$.

\subsubsection{Ensaio a vazio}

Estabilizada a potência de entrada do motor, como é descrito no procedimento para realização do respectivo ensaio na seção 2.5 .3 , foi realizada a medição da tensão, corrente e da própria potência. A Tabela 6 indica os valores de tais medidas obtidas através da fonte programável.

Tabela 6 - Medições obtidas durante o ensaio a vazio

\begin{tabular}{cccc}
\hline Grandeza medida & FASE A & FASE B & FASE C \\
\hline Tensão (Vrms) & 219,97 & 219,97 & 219,97 \\
\hline Corrente $($ Arms) & 4,31 & 4,43 & 4,31 \\
\hline Fase $(\boldsymbol{\phi})$ & 0 & 240,3 & 120,5 \\
\hline Potência $(\mathbf{k W})$ & 0,08 & 0,08 & 0,07 \\
\hline $\begin{array}{c}\text { Potência Aparente } \\
\text { (kVA) }\end{array}$ & 0,547 & 0,563 & 0,547 \\
\hline Fator de Potência & 0,15 & 0,14 & 0,13
\end{tabular}

Fonte: Elaborada pelo autor 
A temperatura registrada no interior do motor foi de $48^{\circ} \mathrm{C}$.

\subsection{Cálculo dos parâmetros do circuito equivalente}

Seguindo o procedimento descrito na seção 2.5.4, parte-se da média aritmética das medidas obtidas nas três fases do motor durante o ensaio de rotor bloqueado, apresentadas na Tabela 7.

Tabela 7 - Média das medições do ensaio de rotor bloqueado

\begin{tabular}{cccc}
\hline & Tensão $(\mathbf{V})$ & Corrente (A) & Potência $(\mathbf{k W})$ \\
\hline Média & 43,3 & $8,52 / 4,92$ & 0,1033 \\
\hline
\end{tabular}

Fonte: Elaborada pelo autor

Substituindo os valores na Equação (3), tem-se o valor da resistência de rotor bloqueado, o qual será igual a:

$$
R_{r t}=\frac{0,1033 \times 1000}{4,92^{2}}=4,270 \Omega
$$

Substituindo os valores na Equação (4), obtém-se o valor da impedância de rotor bloqueado:

$$
Z_{r t}=\frac{43,3}{4,92}=8,800 \Omega
$$

Substituindo os respectivos valores da resistência e da impedância de rotor bloqueado na Equação (5), obtém-se a reatância de rotor bloqueado:

$$
X_{r t}=\sqrt{8,800^{2}-4,270^{2}}=7,694 \Omega
$$

O próximo passo é separar as reatâncias de dispersão tanto do rotor como do estator, da reatância de rotor bloqueado. Para tal, utiliza-se como auxílio o Quadro 2. Verifica-se que a respectiva máquina é da classe $\mathrm{A}$, ou seja, a reatância de dispersão do rotor é igual à do estator. Portanto, seus respectivos valores serão:

$$
X_{1}=X_{2}=\frac{X_{r t}}{2}=3,845
$$

Já que:

$$
X_{r t}=X_{1}+X_{2}
$$

Corrigindo a resistência de rotor bloqueado para uma temperatura de $25^{\circ} \mathrm{C}$, obtém-se um valor de 4,096 $\Omega$, e, posteriormente, substituindo os respectivos valores na Equação (6), 
chega-se finalmente a uma estimativa para o valor da resistência do rotor à temperatura de $25^{\circ} \mathrm{C}$ :

$$
R_{25^{\circ} \mathrm{C}}=R_{r t_{25^{\circ} \mathrm{C}}}-R_{1_{25^{\circ} \mathrm{C}}}=4,096-2,170=1,926 \Omega
$$

Seguindo a metodologia descrita na seção 2.5 .4 , pode-se determinar os parâmetros do ramo magnetizante, partindo-se da média aritmética, apresentada na Tabela 8 , dos valores obtidos durante a medição do ensaio a vazio, os quais estão presentes na Tabela 6 .

Tabela 8 - Médias das medições do ensaio a vazio

\begin{tabular}{ccccc}
\hline & $\begin{array}{c}\text { Tensão } \\
(\mathbf{V})\end{array}$ & $\begin{array}{c}\text { Corrente } \\
(\mathbf{A})\end{array}$ & $\begin{array}{c}\text { Potência } \\
(\mathbf{k W})\end{array}$ & $\begin{array}{c}\text { Fator de } \\
\text { potência }\end{array}$ \\
\hline Média & 219,97 & $4,350 / 2,511$ & 0,076 & 0,14 \\
\hline
\end{tabular}

Fonte: Elaborada pelo autor

Substituindo os respectivos valores na Equação (7), lembrando-se que $R_{1}$ deve ser corrigida para a temperatura do ensaio, que é de $48^{\circ} \mathrm{C}\left(R_{1}\right.$ igual a $\left.2,366 \Omega\right)$. Logo o valor da tensão sobre o ramo magnetizante será:

$$
E_{2}=219,970-\left(\sqrt{(2,366)^{2}+(3,845)^{2}}\right) 2,511=208,636 \mathrm{~V}
$$

Substituindo o ângulo de defasamento entre a tensão e a corrente, cujo valor é de $81,852^{\circ}$, bem como a corrente a vazio, nas Equações (8) e (9), ter-se-á os valores das respectivas correntes descritas na seção 2.5.4.

$$
\begin{gathered}
I_{c}=2,511 x \cos 81,952^{\circ}=351,540 m A \\
I_{m}=2,511 x \operatorname{sen} 81,952^{\circ}=2,486 A
\end{gathered}
$$

Por fim, por meio da Equação (1), é determinado o valor da resistência e da reatância do ramo magnetizante.

$$
\begin{gathered}
R_{m}=\frac{E_{2}}{I_{c}}=\frac{208,636}{351,510 m A}=593,491 \Omega \\
X_{m}=\frac{E_{2}}{I_{m}}=\frac{208,636}{2,486}=83,924 \Omega
\end{gathered}
$$




\subsection{Simulação computacional}

A simulação computacional do motor visa analisar o comportamento do mesmo em regime permanente, desconsiderando portanto, o regime transitório. Sendo assim, o método de partida não irá interferir no estudo a ser realizado. Logo, optou-se pela partida direta.

Todas as simulações foram realizadas no PSIM $®$, a partir do circuito apresentado pela Figura 6.

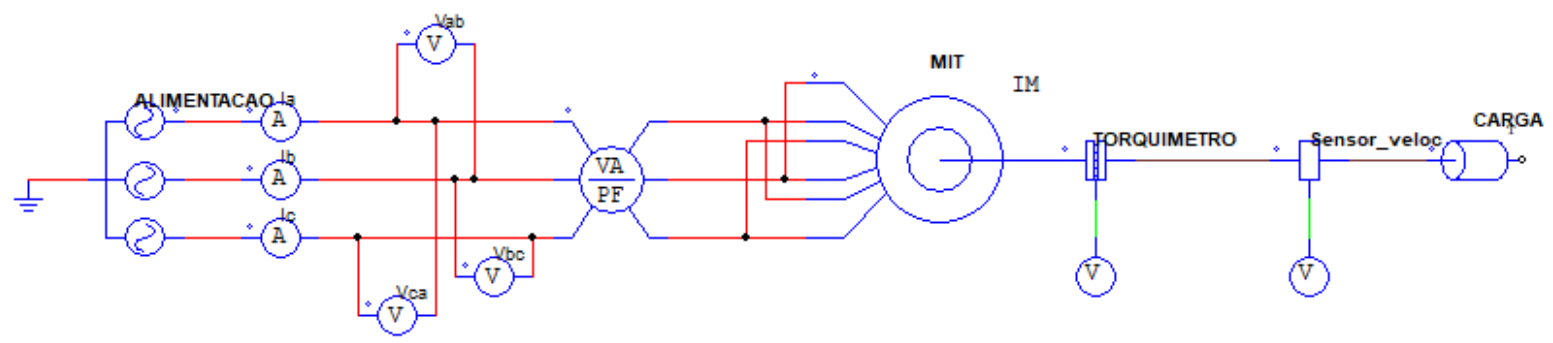

Figura 6 - Circuito realizado no PSIM® para a simulação Fonte: Elaborada pelo autor

Foi realizado o ensaio com variação de carga, utilizando, para isso, uma máquina de corrente contínua acoplada ao eixo do motor de indução. Tal procedimento possui como objetivo servir de comparação com a simulação realizada. Os valores obtidos estão apresentados na Tabela 9.

Tabela 9 - Medições do ensaio com variação de carga

\begin{tabular}{ccccc}
\hline $\begin{array}{c}\text { Carga com } \\
\text { relação a } \\
\text { corrente nominal } \\
\text { do MIT(\%) }\end{array}$ & FASE A & FASE B & FASE C & Média \\
\hline $100 \%$ & 0,86 & 0,86 & 0,87 & 0,863 \\
$75 \%$ & 0,82 & 0,81 & 0,82 & 0,816 \\
\hline
\end{tabular}

Fonte: Elaborada pelo autor

Os parâmetros do circuito equivalente utilizados na simulação para o motor de indução trifásico estão apresentados no Quadro 3. Tais valores referem-se à aqueles obtidos no item 3.1, contudo, referidos à temperatura de $101^{\circ} \mathrm{C}$, a qual foi registrada durante a variação da carga. 


\begin{tabular}{|c|c|}
\hline \multicolumn{2}{|c|}{ Parâmetros do circuito equivalente } \\
\hline Resistência do estator & $2,811 \Omega$ \\
\hline Resistência do rotor & $2,503 \Omega$ \\
\hline Reatância do estator & $3,845 \Omega$ \\
\hline Reatância do rotor & $3,845 \Omega$ \\
\hline Reatância de magnetização & $83,966 \Omega$ \\
\hline
\end{tabular}

Quadro 4 - Parâmetros do circuito equivalente à $101^{\circ} \mathrm{C}$

Fonte: Elaborada pelo autor

A primeira análise foi realizada considerando carga nominal, a qual pode ser calculada por meio da Equação (10).

$$
T=\frac{P}{W}
$$

Na qual:

- $T$-Torque (N.m)

- $\quad w$-Velocidade $(\mathrm{rad} / \mathrm{s})$;

- $P$ - Potência (watts);

Sendo a potência do motor igual a $2200 \mathrm{~W}$ e a velocidade nominal igual a $3450 \mathrm{rpm}$, ao substituir na Equação (10), obtém-se o valor do torque nominal, que será utilizado no dimensionamento da carga.

$$
T=\frac{2200}{\frac{\pi}{30} \times 3450}=6,089 \mathrm{~N} . \mathrm{m}
$$

O presente trabalho visou a análise do fator de potência para dois pontos de cargas, como pode ser vizualizado através da Tabela 9. Para o primeiro ponto, a carga foi ajustada de tal forma que o motor trabalhasse com carga nominal. A Figura 7 representa o fator de potência obtido para o respectivo ponto.

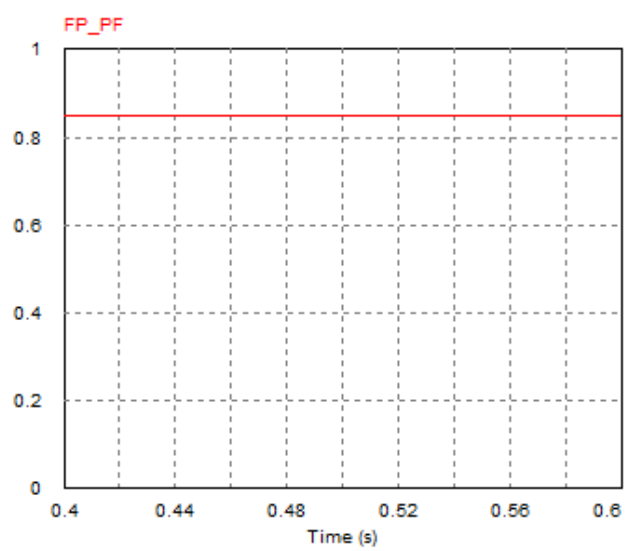

Figura 7 - Fator de potência x Tempo (s) 100\%/0,850

Fonte: Elaborada pelo autor.

ForSci.: r. cient. IFMG campus Formiga, Formiga, v. 7, n. 1, e00362, jan./jun. 2019. 
O fator de potência da simulação resultou em um valor de 0,850 , que, comparado com o valor médio de 0,863 , obtido durante o ensaio experimental, presente na Tabela 9, evidencia a coerência e precisão dos resultados teóricos.

Para corroborar melhor os resultados, foi analisado outro ponto de carga, como está apresentado na Tabela 9, cujo valor é de $75 \%$ em relação a carga nominal.

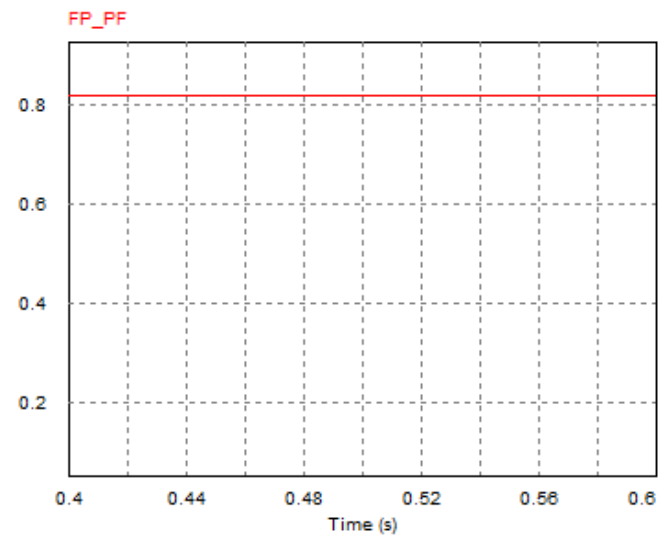

Figura 8 - Fator de potência x Tempo (s) 75\% / 0,819 Fonte: Elaborada pelo autor.

A Figura 8 representa o fator de potência obtido na simulação para $75 \%$ de carga. $O$ valor do fator de potência registrado para este ponto de carga durante a simulação foi de 0,819, que, comparado com o valor médio de 0,816 , obtido durante o ensaio, apresentado na Tabela 9, indica a confiabilidade dos resultados obtidos com a simulação.

A redução da carga ou o superdimensionamento dos motores pode influenciar na queda do fator de potência de um sistema elétrico, como pôde ser verificado anteriormente durante a realização dos experimentos e simulações. Quando se considera uma indústria, onde este tipo de equipamento geralmente representa a maior parte de sua carga elétrica, como está representado por meio da Figura 9, este problema torna-se significativo.

\section{Consumo Energia Elétrica Indústria - GWh}

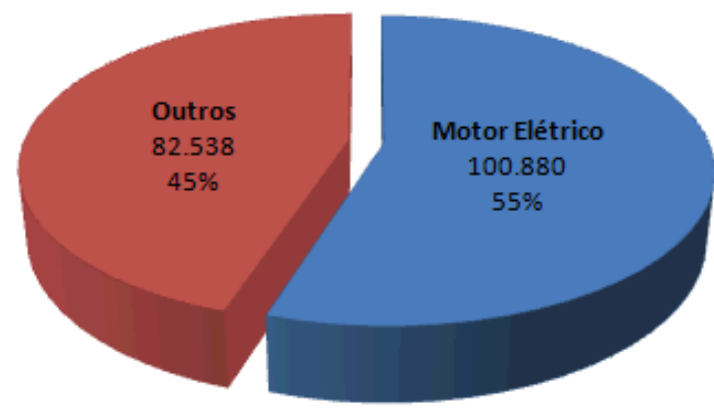

Figura 9 - Consumo de energia nas indústrias Fonte: Weg (2009).

ForSci.: r. cient. IFMG campus Formiga, Formiga, v. 7, n. 1, e00362, jan./jun. 2019. 
Considerando-se um sistema de três motores dimensionados com os parâmetros do Quadro 3 operando com $100 \%$ de carga nominal, e outro sistema com os mesmos motores, operando com, respectivamente, $75 \%, 65 \%$ e $50 \%$ de carga, tem se os fatores de potência representados pelas Figuras 11 e 12, respectivamente.

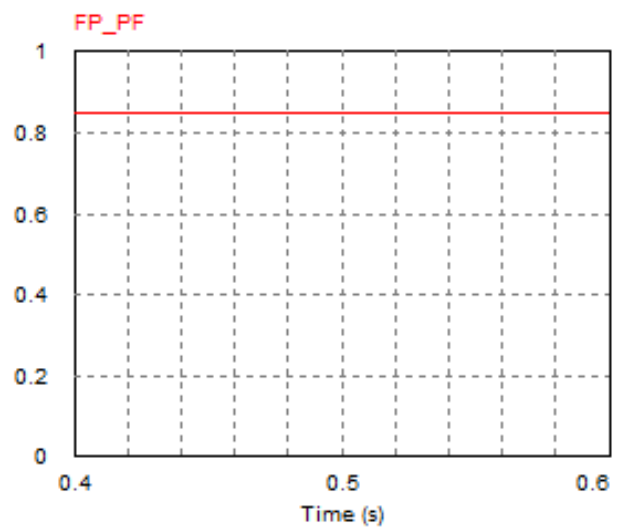

Figura 7 - Fator de potência x Tempo (s) - Sistema com três motores a 100\% de carga Fonte: Elaborada pelo autor.

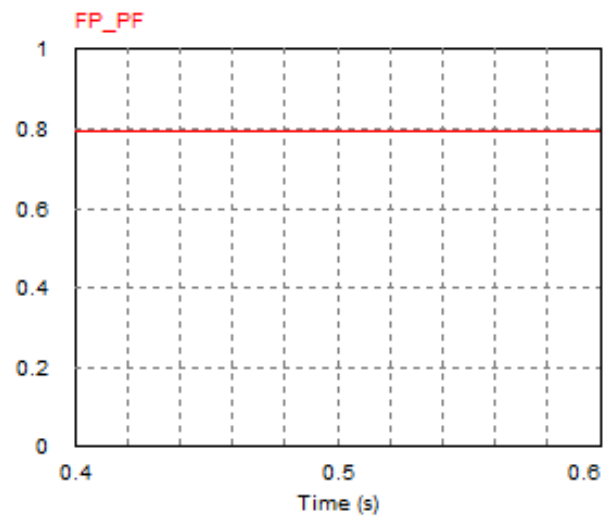

Figura 8 - Fator de potência x Tempo (s) - Sistema com três motores a 75\%,65\% e 50\% de carga Fonte: Elaborada pelo autor.

Para o sistema operando com $100 \%$ de carga, o fator de potência registrado foi de 0,851 e para o sistema operando com $75 \%, 65 \%$ e $50 \%$, o fator de potência registrado foi de 0,795 .

Tendo em vista que a quantidade de motores em uma indústria pode ser bem maior que a quantidade de motores utilizados nos sistemas acima, é possível constatar a acentuada queda do fator de potência com o superdimensionamento de motores.

O baixo fator de potência pode acarretar multas, como é estabelecido pela Agência Nacional de Energia Elétrica através do Artigo nº 95 da Resolução ANEEL nº414 de 09 de 
setembro de 2010, devido ao consumo excessivo de potência reativa. Nos casos em que o fator de potência não contemple o valore indicado na norma, é necessário realizar a correção do fator de potência através do emprego de banco de capacitores. Sendo assim, o dimensionamento adequado dos motores de indução trifásicos acaba evitando este problema e, consequentemente, os gastos desnecessários.

\title{
4 CONCLUSÃO
}

Seguir as recomendações da norma possibilitou a realização dos ensaios de forma prática e segura. Consequentemente, as medições para os cálculos dos parâmetros do circuito equivalente do motor de indução trifásico, contribuíram decisivamente para a obtenção dos parâmetros de forma mais exata possível.

Por meio das simulações realizadas, pôde ser verificado que os parâmetros do motor de indução trifásico, obtidos por procedimentos dispostos na norma NBR17094-3:2018, mostraram-se coerentes, devido ao fato dos resultados das simulações terem se aproximado dos resultados dos experimentos realizados com o motor.

A análise de motores superdimensionados permite verificar o quão importante é realizar o dimensionamento adequado de tal máquina de acordo com a carga que será acoplada ao seu eixo, tendo em vista a influência direta sobre o fator de potência, onde o mesmo decai sempre que houver uma carga menor que a nominal, como pôde ser verificado.

\section{ANALYSIS OF THE PERFORMANCE OF THE THREE-PHASE INDUCTION MOTOR THROUGH TESTS RECOMMENDED IN TECHNICAL STANDARD AND COMPUTATIONAL SIMULATION}

\begin{abstract}
This work presents a study carried out to determine the parameters of the equivalent circuit of a three-phase induction motor based on the tests recommended in the NBR17094-3:2018 Brazilian Association of Technical Standards - in addition to the verification of its performance through the variation of load on its axis, using computational simulation. The parameters obtained during the tests are used in software for the analysis of the power factor in different load situations.
\end{abstract}

Keywords: Three phase induction motor. NBR17094-3:2018. Parameters. Computational Simulation. 


\section{REFERÊNCIAS}

ASSOCIAÇÃO BRASILEIRA DE NORMAS TÉCNICAS. NBR17094-3:2018: máquinas elétricas girantes. Parte 3: Motores de indução trifásicos: métodos de ensaio. Rio de Janeiro, 2018.

DA SILVEIRA, F. L.; MARQUES, N. L. R. Motor elétrico de indução: uma das dez maiores invenções de todos os tempos. Caderno Brasileiro de Ensino de Física, São Paulo, v. 29, n. 1, p. 114-129, 2012.

FITZGERALD, A. E. et al. Máquinas Elétricas: com introdução a eletrônica de potência. Tradução: Anatólio Laschuk. 6. ed. Porto Alegre: Bookman, 2006.

RESOLUÇÃO ANEEL N414 DE 09 DE SETEMBRO DE 2010. Disponível em: http://www.aneel.gov.br/documents/656877/14486448/bren2010414.pdf/3bd33297-26f94ddf-94c3-f01d76d6f14a? Version=1.0. Acesso em: 1 fev. 2018.

WEG. Oportunidades em eficiência energética. 2009. Disponível em: http://www.weg.net/institutional/BR/pt/news/geral/oportunidades-em-eficiencia-energetica. Acesso em: 01 mar. 2018.

\section{DADOS DOS AUTORES}

\section{Vinicius Marcos Pinheiro}

E-mail: viniciusmarcospinheiro@ hotmail.com

Currículo Lattes: http://lattes.cnpq.br/8748537162782615

Graduação em Engenharia Elétrica pela Universidade Federal de Uberlândia.

\section{Luciano Coutinho Gomes}

E-mail: lcgomes@ufu.br

Currículo Lattes: http://lattes.cnpq.br/3372531394380706

Doutorado e graduação em Engenharia Elétrica pela Universidade Federal de Uberlândia (UFU). Atualmente é professor Associado I da Faculdade de Engenharia Elétrica da UFU, tendo sido Coordenador de seu Curso de Graduação em Engenharia Elétrica de 2013 a 2017. De 2015 a 2018, foi Coordenador Geral de um Projeto Brafitec (intercâmbio entre alunos franceses e brasileiros). Tem experiência na área de Acionamentos Elétricos, Processamento Digital de Sinais, Máquinas e Acionamentos Elétricos. Atua, principalmente, no estudo de técnicas de acionamento digital, e no desenvolvimento e projeto de máquinas elétricas

\section{Cássio Alves de Oliveira}

E-mail: kass-07@hotmail.com

Currículo Lattes: http://lattes.cnpq.br/6920155455622196

Mestrado em Engenharia Elétrica (linha de pesquisa: Acionamentos de Máquinas Elétricas) pela Universidade Federal de Uberlândia (UFU) e graduação em Engenharia Elétrica com certificação em Engenharia de Sistemas de Energia Elétrica pela UFU. 